\title{
Bu open A qualitative study of patients' perceptions and priorities when living with primary frozen shoulder
}

\author{
Susan Jones, ${ }^{1}$ Nigel Hanchard, ${ }^{1}$ Sharon Hamilton, ${ }^{1}$ Amar Rangan ${ }^{2}$
}

To cite: Jones S, Hanchard N, Hamilton S, et al. A qualitative study of patients' perceptions and priorities when living with primary frozen shoulder. BMJ Open 2013;3:e003452. doi:10.1136/bmjopen-2013003452

- Prepublication history for this paper is available online. To view these files please visit the journal online (http://dx.doi.org/10.1136/ bmjopen-2013-003452).

Received 21 June 2013 Revised 16 August 2013 Accepted 23 August 2013

${ }^{1}$ Health and Social Care Institute, Teesside University, Middlesbrough, UK ${ }^{2}$ Department of Orthopaedics, The James Cook University Hospital, Middlesbrough, UK

Correspondence to Nigel Hanchard; N.Hanchard@tees.ac.uk

\section{ABSTRACT}

Objectives: To elucidate the experiences and perceptions of people living with primary frozen shoulder and their priorities for treatment.

Design: Qualitative study design using semistructured interviews.

Setting: General practitioner (GP) and musculoskeletal clinics in primary and secondary care in one National Health Service Trust in England.

Participants: 12 patients diagnosed with primary frozen shoulder were purposively recruited from a GP's surgery, community clinics and hospital clinics. Recruitment targeted the phases of frozen shoulder: pain predominant $(n=5)$, stiffness predominant $(n=4)$ and residual stiffness predominant following hospital treatment $(n=2)$. One participant dropped out. Inclusion criteria: adult, male and female patients of any age, attending the clinics, who had been diagnosed with primary frozen shoulder.

Results: The most important experiential themes identified by participants were: pain which was severe as well as inexplicable; inconvenience/disability arising from increasing restriction of movement (due to pain initially, gradually giving way to stiffness); confusion/ anxiety associated with delay in diagnosis and uncertainty about the implications for the future; and treatment-related aspects. Participants not directly referred to a specialist (whether physiotherapist, physician or surgeon) wanted a faster, better-defined care pathway. Specialist consultation brought more definitive diagnosis, relief from anxiety and usually selfrated improvement. The main treatment priority was improved function, though there was recognition that this might be facilitated by relief of pain or stiffness. There was a general lack of information from clinicians about the condition with over-reliance on verbal communication and very little written information.

Conclusions: Awareness of frozen shoulder should be increased among non-specialists and the best available information made accessible for patients. Our results also highlight the importance of patient participation in frozen shoulder research.

\section{INTRODUCTION}

Frozen shoulder (FS) is a condition affecting the capsule of the shoulder joint and is

\section{ARTICLE SUMMARY}

Strengths and limitations of this study

- This study is the first to focus on patients' experiences of conventional care pathways for frozen shoulder (FS) and their priorities for treatment.

- Patients were recruited from care settings where the condition is typically managed. The number of participants was small, so the findings must be considered indicative rather than conclusive, but enough repetition took place to be confident in the themes identified. In addition, two participants' experience of FS was retrospective. Compared to recent cases, their contributions are likely to be more open to error of memory and to reflect less current approaches to care.

- Nevertheless the design included trustworthy methods with clear reporting, allowing readers to make their own judgement on trustworthiness. As the work published on this topic is very limited this article makes a valuable contribution.

characterised by inflammation and contracture. ${ }^{1}$ These events may occur for no identifiable reason in 'primary' or 'idiopathic' FS. 'Secondary' FS is associated with some other event or condition: the most common associated event is trauma, ${ }^{2}$ whereas associated conditions include rotator cuff disease, hemiparesis and others. ${ }^{3}$ Diabetes is a known risk factor for $\mathrm{FS}^{4}{ }^{4}$ Some authorities consider FS associated with diabetes to be a 'primary' type and others consider it 'secondary. 5

FS affects around $10 \%$ of the general adult population, ${ }^{6}$ men and women approximately equally, ${ }^{6} 7$ and the prevalence of those seeking help is $2-5 \% .^{8}$ To our knowledge, there are no published data on the relative prevalence of primary and secondary FS. However, FS of no detectable cause is thought to predominate, with patients whose FS is associated with diabetes constituting an additional 'substantial' group," accounting for almost one-third of all FS.

The healthcare implications of FS are considerable. In the UK, for example, based on 
a single general practitioner (GP) consultation for each case, it costs the National Health Service (NHS) at least $£ 44.1$ million (assuming a prevalence of $2 \%$ ) or $£ 110.3$ million (assuming a prevalence of 5\%). And since the usual age at onset is $40-60$ years, ${ }^{9}$ which is typically within the working-age range, there is a crucial economic impact on individuals and society.

Codman, who coined the term frozen shoulder, described the typical clinical manifestation as early as 1934

The condition [comes] on slowly; pain usually felt near the insertion of the deltoid; inability to sleep on the affected side; painful and incomplete elevation and external rotation; restriction of both spasmodic and mildly adherent type; atrophy of the spinati; little local tenderness; X-rays negative except for bone atrophy. ${ }^{10}$

However, the location of pain may be variable ${ }^{9}$ and diagnosis may present a challenge, particularly for the non-specialist and particularly in the early stages, when the signs are not pronounced: not least because falsepositive responses to standard tests for shoulder impingement (Neer's sign, Hawkins' test) are to be expected. $^{11}$

Recognising FS as phasic in nature, the recent UK guidelines for diagnosis, assessment and physiotherapy management of FS recommend a simple dichotomous 'pain predominant' or 'stiffness predominant' classification, whereby the patient is the arbiter of the stage and pain takes precedence when there is ambiguity. ${ }^{11}$ Potential interventions include watchful waiting, physiotherapy, steroid injection, distension arthrography, manipulation under anaesthetic (MUA) and arthroscopic capsular release. ${ }^{5}{ }^{11}$ There is some evidence for specific interventions, although none of this is strong ${ }^{6} 12$ and not all observational series have verified the certainty of complete recovery. For example, in a recent study of 223 patients with FS referred to tertiary care, $38 \%$ had persistent mild symptoms, mostly pain, at a mean follow-up of 4.4 years (range 2-20 years). In 3\% the persistent symptoms were severe, with pain and functional deficits. ${ }^{13}$

Despite a general appreciation that the condition imposes a serious burden on sufferers, the research literature demonstrates a lack of interest in the subjective experience of FS and patients' priorities for treatment. A recent systematic review commissioned by the UK Department of Health sought studies on patients' views of conventional treatments, but found none. ${ }^{5}$ A similar search without restriction on treatment type revealed just one study, focused on Bowen therapy and providing only limited insights. ${ }^{14}$ This paucity is surprising, as there is evidence to show that patients' attitude towards treatment significantly affects concordance, including in musculoskeletal (MSK) conditions. ${ }^{15}$ Therefore, it is important to consider the patient perspective when assessing overall treatment effectiveness. ${ }^{15}$ This has become increasingly relevant with the move towards a patient-centred paradigm of healthcare. ${ }^{15}$ This paper seeks to qualitatively explore the experiences and perceptions of people living with primary frozen shoulder and their priorities for treatment.

\section{METHODS}

A qualitative study design employing semistructured interviews was used to explore the experiences, priorities and perceptions of those experiencing the different phases of primary FS. Primary FS was chosen as the inclusion of secondary FS might have resulted in excessive heterogeneity.

\section{Participants \\ Inclusion criteria}

Adult, male and female patients of any age, with or without diabetes, who had been diagnosed with primary FS by the following criteria:

- Shoulder pain for at least 1 month;

- Reduction in passive external rotation of $50 \%$ or more compared to the contralateral side ${ }^{16}$ with a clinically significant change in end-feel;

- No clinical suspicion of other pathologies that might present similarly;

- Normal X-rays (only if clinically indicated).

\section{Recruitment}

Recruitment occurred in community and hospital settings. Clinical members of the research team who recruited participants from the community worked in a general medical practice; however, in addition to their own surgery clinics they also recruited from clinics that they ran in collaboration with other specialist doctors and physiotherapists, which were spread across the community. The patients recruited from the MSK and hospital-based clinics had been referred to specialist services.

\section{Sampling}

A structured, purposive sample of patients in the different phases of $\mathrm{FS},{ }^{17}$ as defined by the UK guidelines, ${ }^{11}$ with a story to tell, ${ }^{18}$ was selected by the clinicians. Group 1 comprised of patients in the pain predominant phase and was drawn exclusively from community care; group 2 were in the stiffness predominant phase, drawn from community as well as secondary care (ie, the hospital setting) and group 3 were in the residual stiffness predominant phase following hospital treatment and drawn exclusively from secondary care. Clinicians were given a standard protocol to use, which provided basic details of the study and what would be required of participants. Patients were given an introductory pack which included an information sheet and contact details, requesting that they respond directly to the research team. When a potential participant contacted the team they were given the opportunity to ask any questions 
prior to arranging an interview. Twenty-two packs were handed out and 12 patients responded; none were excluded. The demographic characteristics of the sample were consistent with the FS population across the UK at large. ${ }^{67}$

\section{Data collection}

Interviews took place on NHS or University premises or by telephone. Each started by establishing informed consent. Interviewees were then allowed the opportunity to give detailed descriptions of their experiences, with individual interviews lasting approximately 30-45 min. Schedules comprising the topics to be covered and a range of prompts relating to specific issues of interest were used to guide data collection during interviews. Questions not only related to the particular phase currently experienced by participants but also included questions about the previous phase, where applicable. The interviews mainly comprised of open questions to encourage the participants to tell their stories. Field notes were made of observations to supplement the transcripts. Credibility was demonstrated by confirmation of information from multiple participants leading to identification of common themes. Interviews were audio recorded with the permission of participants and later transcribed. Interviews, transcription and subsequent data checking were undertaken by healthcare researchers experienced in qualitative research ( $\mathrm{SJ}$ and $\mathrm{SH}$ ).

\section{Data analysis}

An inductive approach to analysis was taken, using a constant comparative method. Data were analysed after each interview and the findings formed the questions and topics for subsequent interviews. The data were independently analysed by two researchers using the six-stage thematic approach outlined by Braun and Clarke. ${ }^{19}$ An in-depth familiarisation with the data was followed by the generation of initial codes, using Nvivo V.9, which were then applied to the data and collated into potential themes independently by the researchers. These themes were then reviewed, generating a thematic map of the analysis. In the next stage the themes were refined and named. Finally, examples were selected to illustrate the thematic framework.

\section{RESULTS}

Participants

Data collection occurred between July 2011 and November 2011. Twelve participants were recruited, and 11 agreed to be interviewed. Five met the criteria for group 1, four for group 2 and two for group 3 (see table $1)$. They included six women and five men, aged from 40 years and above, predominantly in their 50s $(n=7)$. Three of the men had diabetes and formed the younger end of the group, with two in their 40s and one in his early 50s. Three participants had experienced FS in both shoulders (though none on both sides concurrently). One participant may have experienced FS in both shoulders, but could not remember the diagnosis given for the first-affected shoulder. One participant, with a previous history of impingement (not FS) in the contralateral shoulder, was initially given the same diagnosis on the currently affected side, but was subsequently diagnosed with FS. Two of the participants had suffered with FS several years ago and were recruited as their history was known to the physiotherapist.

\section{Contexts of participant journeys}

One participant consulted a GP 1 week after the start of symptoms but all the others waited $6-8$ weeks before presenting for a consultation. After initial presentation they followed various paths towards specialist treatment. Some participants experienced delay in receiving a definitive diagnosis and specialist (ie, MSK or shoulder specialist) care (see table 1).

\section{Thematic analysis}

Four major patient-reported themes associated with FS were identified and are shown in the thematic map (figure 1).

\section{Theme 1: pain}

Patients reported that the pain would start quite suddenly and gradually worsen over weeks and months. During the initial (pain predominant) phase the pain would be very intense on certain movements, typically stretching or reaching in specific directions. Unguarded movement, in particular, caused severe pain. Also, sleep was badly disturbed. Once awake, participants struggled to get back to sleep due to constant, 'nagging' pain. Sleep deprivation wore participants down, even though they reported taking a resilient approach. In an attempt to manage their pain, participants tried to avoid painful movements, applied ointments and took medication. The nature of the pain was such that they worried about what could be the cause.

The amount of pain that I was in, I didn't believe that it was just a frozen shoulder. Participant 12

Finding a way to deal with the pain and carry on with life as normally as possible was very important to participants.

\section{Theme 2: awareness and expectations}

Participants did not successfully self-diagnose their problem. Typically they thought they had 'pulled something', anticipating that it would resolve in a week or two. Diagnosis also presented a challenge among nonspecialist healthcare staff.

I made an appointment at the doctors and the doctor, having examined me, said, 'you are lucky it isn't a frozen shoulder, I think that what you have done is you have trapped a nerve, you have some slight nerve damage, you 
Table 1 Participants' characteristics

\begin{tabular}{|c|c|c|c|c|c|c|c|c|}
\hline $\begin{array}{l}\text { Participant } \\
\text { number }\end{array}$ & $\begin{array}{l}\text { Age in } \\
\text { years }\end{array}$ & Gender & Diabetic & Group & $\begin{array}{l}\text { Dominance } \\
\text { of affected } \\
\text { side at time } \\
\text { of study }\end{array}$ & $\begin{array}{l}\text { Length of time } \\
\text { from onset to } \\
\text { first consultation }\end{array}$ & $\begin{array}{l}\text { Length of time from } \\
\text { first consultation to } \\
\text { referral to } \\
\text { musculoskeletal } \\
\text { (MSK) specialist }\end{array}$ & Where physiotherapy treatment was given \\
\hline 1 & $60-69$ & Female & No & 1 & $\mathrm{D}$ & Within 3 months & 3 months & Own practice-physiotherapist with MSK interest \\
\hline 2 & $50-59$ & Female & No & $1 \& 2$ & ND \& D & $\begin{array}{l}\text { Immediate } \\
\text { Within } 2 \text { months }\end{array}$ & $\begin{array}{l}\text { Immediate } \\
22 \text { months }\end{array}$ & $\begin{array}{l}\text { MSK community clinic } \\
\text { GP at own practice (not referred to MSK specialist) }\end{array}$ \\
\hline 3 & $50-59$ & Female & No & 1 & $\mathrm{D}$ & Within 2 months & 0 months & Own practice-physiotherapist with MSK interest \\
\hline 4 & $50-59$ & Female & No & 2 & ND & Unknown & Unknown & Own practice physiotherapist (not referred to MSK specialist) \\
\hline 5 & $50-59$ & Male & No & 2 & $\mathrm{D}$ & Within 1 month & 5 months & Hospital MSK clinic \\
\hline 6 & $40-49$ & Male & Yes & 2 & $\mathrm{D}$ & Within 2 months & 3 months & $\begin{array}{l}\text { Occupational health physiotherapist and own practice- } \\
\text { physiotherapist with MSK interest }\end{array}$ \\
\hline 7 & $50-59$ & Female & No & 1 & ND & Approx 5 months & 0 months & MSK community clinic \\
\hline 8 & Over 70 & Male & No & 1 & $\mathrm{D}$ & Within 3 months & 0 months & Own practice—physiotherapist with MSK interest \\
\hline 10 & $50-59$ & Female & No & 2 & ND & Within 2 months & 6 months & Generalist physiotherapist and MSK hospital clinic \\
\hline 11 & $50-59$ & Male & Yes & 3 & ND & Within 2 months & 8 months & MSK hospital clinic \\
\hline 12 & $50-59$ & Male & Yes & 3 & ND & Within 1 month & 16 months & $\begin{array}{l}\text { Occupational health physiotherapist and MSK } \\
\text { hospital clinic }\end{array}$ \\
\hline
\end{tabular}


Figure 1 Thematic map.

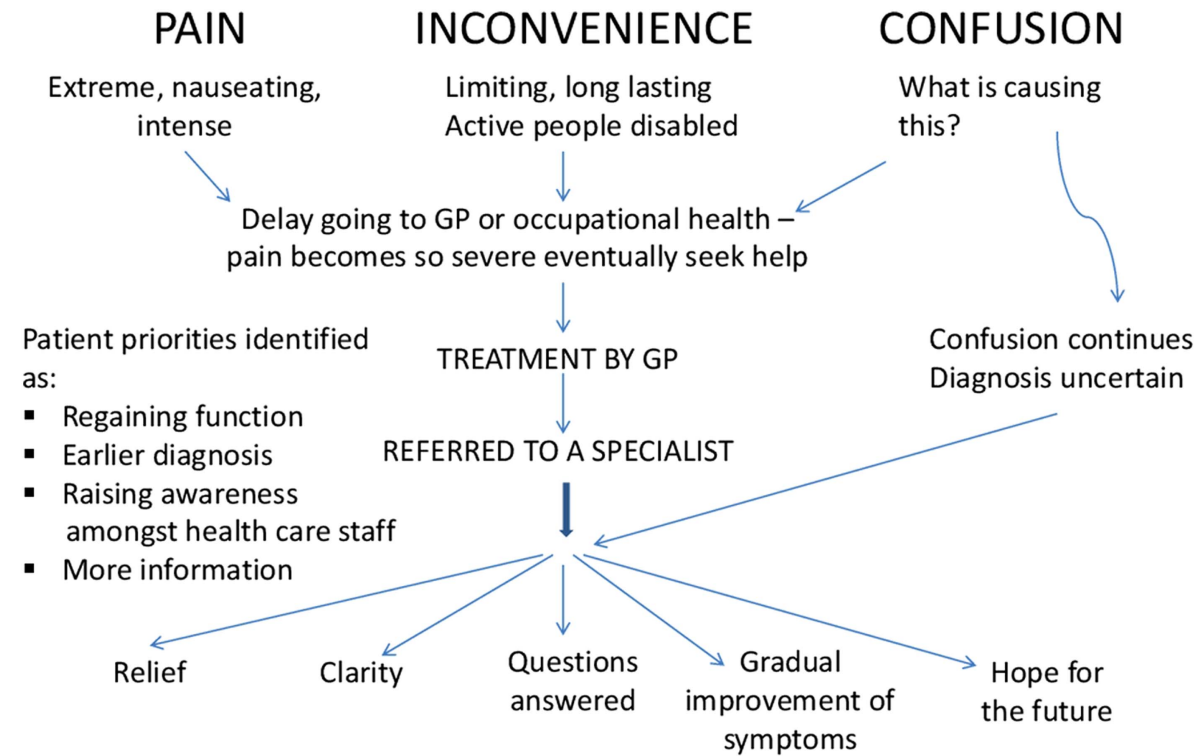

just need to rest, it will take a couple of months.'

Participant 5

Lack of diagnosis or misdiagnosis led to diverse consequences among the participants; for example, anxiety, denial and delays in definitive diagnosis and referral. Participants were often left with unanswered questions, uncertainty as to the treatment options and the potential risks and benefits of treatment, occasionally, with contradictory advice.

... you want to know where the final outcome is going to be rather than you suffering week in week out thinking, 'when is it going to end? Fed up with this.' Whereas when you know it is going to last 3 or 4 years down the line, I know it is not going to be forever. Whereas you don't know anything; God, is this ever going to go away? I think I would like to know from the start. Participant 11

I don't know whether I wasn't saying the right things to [my GP] or what... I don't know whether it is my relationship with the doctor or what, but I don't ... I'm not very good, if they are vague and I don't understand what they are talking about, I won't sit there any longer, I'll, I'll leave. All I wanted was an answer. Participant 12

Participants who did not receive early informed or specialist care would have preferred a quick, clear pathway and although they understood the difficulty of diagnosis, some expressed disappointment that they had suffered, for what seemed to them, longer than necessary, due to a lack of awareness of the condition on the part of their healthcare providers.

With the shoulder, I was saying, 'it's still hurting' and they [the GPs] were saying 'we'll look to see what else it is then.' Every time, something came back as, 'no, it's negative. Have you got any neck problem, shoulder problem, anything like that?' No arthritis, no swelling in it ... but every time it come back no, you are thinking, now what? Participant 7 [first-affected shoulder, 2004]

\section{Theme 3: inconvenience/disability}

Typically the FS lasted from 1 to 3 years. In a group of people who were usually active, this was hugely disruptive. The inability to use the arm normally, initially due to pain, gradually giving way to stiffness, affected even very fundamental activities of daily living (ADL), including, among others, hair washing and styling, getting out of a bath, dressing, reaching into a back pocket, opening a door, pegging washing out, washing up and other housework, home maintenance jobs, lifting a kettle, cutting up food, pulling a suitcase or wheeled shopping basket, gardening and, when driving, changing gear or positioning for reversing. In order to continue to drive, one participant had to buy a car with automatic transmission.

Try getting out the bath 'cause you can't find a way up. You just cannot find a way to get out the bath! We've got bars on the side of the bath anyway but I couldn't find a way. I just had to snake over the side sometimes. Participant 7

Participants who worked had to change their work routines to accommodate their limitations; one, for whom this was not possible, had to resign.

A general observation was that the pain and these major impairments in ADL occurred without obvious outward signs, so that much of the suffering involved was hidden from the casual observer. This led to much less sympathy than would be the case with many conditions.

I was still doing the workload as such, so you don't get any, it's awful to say, you don't get any sympathy from people because it is not something that stops you doing things but in yourself you feel so frustrated, because when I reached up to do anything, we do a lot of 
overhead stuff, there was no strength in the arm, it was very painful but nobody would say, "How is it?" You felt that they didn't understand, because you are at work with it and that was the hardest thing, and they still expected you to do things because you didn't have your arm strapped up. Participant 10

A further cause of hidden suffering was that other people relied on their own experiences of pain when trying to understand the participants' perspective but it was outside their lexicon of knowledge. Three participants recognised that they themselves had not understood what it was like or been sufficiently sympathetic towards others with FS until they acquired it themselves.

I've heard of other people with frozen shoulders and I'm afraid I haven't given them much sympathy because I didn't know what it involved. You would be in the same boat. I sympathised, say that's terrible, but really unless you have or have had a frozen shoulder you don't know how painful it is. It is really excruciating. Participant 8

In general, so long as the FS was seen as temporary and benign and they could care for themselves and keep their jobs until they recovered, participants accepted their situation; at the same time, they hoped for the earliest possible resolution.

\section{Theme 4: treatment}

Participants identified functional outcomes, for example, freedom of movement, as their main priority from treatment; however, they recognised that if the pain or stiffness remained they would be unable to recover the movement. On presentation at the GP's surgery, reflecting the lack of a definitive diagnosis in most cases, there was no standard course of treatment offered. Two participants came through their occupational health physiotherapy services then to their GPs. Neither of them received a diagnosis of FS either. Some GPs opted to wait and see and most prescribed analgesics. As time passed some offered further investigations and/or administered further first-line treatment themselves, while others referred patients to an on-site physiotherapist or to a primary or secondary care MSK clinic.

The range of first-line treatments experienced by the participants from their GPs included ointment, medications and local injections. On referral to the physiotherapist, treatment might include advice and education, hands-on passive mobilisation, exercises, local injections, heat, massage, ultrasound, transcutaneous electrical nerve stimulation, acupuncture or hydrotherapy.

All participants had received some physiotherapy due in part to the recruitment pathways into the study. Some participants were referred swiftly to a specialist physiotherapist, a factor which may have been influenced by the involvement of a surgery with a physiotherapist specialising in MSK and FS. In other cases participants were referred, only after lengthy periods of care by an occupational physiotherapist and/or their own GP, to a specialist physiotherapist, a GP with a special interest in MSK conditions or a consultant shoulder and elbow surgeon. Two participants received surgical interventions: MUA and an operative capsular release, respectively.

MSK specialists, whether specialist physiotherapists, GPs with a special interest or surgeons, were available in various locations. There were sometimes delays in referral to these specialists, but once referred, participants reported positively on their care in each location. Definitive diagnoses were made or confirmed. Referral also brought an understanding approach, clarity and relief; participants expressed profound relief at knowing what the problem was and being in the care of someone who knew about the condition. Some of their worries could be explained and their questions answered. This was very important to them.

[S/he] went into extreme [detail], were there any questions, [s/he] was so incredibly thorough, I can't say enough really. $[\mathrm{S} / \mathrm{he}]$ went into, straight away what it was, because what I couldn't understand was, why was I getting this pain in the top of my arm when it is a frozen shoulder, but why am I getting pain in the arm and not the shoulder?

What did [s/he] say?

[S/he] did me a diagram; [s/he] showed me a model of a skeleton.

\section{Participant 10}

Relationships with specialists were generally reported as friendly and non-hierarchical and conducive to the exchange of information. In general, however, there was over-reliance on verbal information, with very limited use of other media.

\section{DISCUSSION}

\section{Principal findings}

Patients' initial experience was characterised by severe, inexplicable pain followed by increasing restriction of movement. Overcoming these symptoms and recovering functional capacity was their priority. Understanding the cause, seriousness and likely outcome of the condition were also important to them. Reducing delay in diagnosis was a common plea with considerable anxiety, confusion and delay for those in the continued care of non-specialists. This was followed by a sense of relief on meeting a specialist, with gradual improvement ensuing in most cases. Most patients found hope and encouragement through this interaction, although elements of the condition remained inexplicable and uncertain. There was over-reliance on verbal communication and very little written information was provided.

\section{Strengths and weaknesses of the study}

This study is the first to focus on patients' experiences of conventional care pathways for this condition and 
their priorities for treatment. These aspects and the in-depth nature of our analysis, are major strengths.

Patients were recruited from care settings where the condition is typically managed; however, some barriers to recruitment were experienced which have been identified previously, such as an initial hiatus in diagnosing the condition and approaching potential participants within a busy clinical setting. Recruitment issues are not uncommon and are well documented in other studies. ${ }^{20}$ This was a small study which consequentially included data from a limited number of patients from a single geographical area. Although their own stories remain valid every possible theme would not necessarily be exhausted. ${ }^{21}$ In addition two participants' experiences of FS was historical, so that, compared to recent cases, their contributions are likely to be more open to error of memory and to reflect earlier approaches to care. Nevertheless the design included trustworthy methods such as using a standard protocol to introduce the study to prospective participants, selecting patients at different stages in the disease trajectory, using a topic schedule for interviews and recording interviews for transcription.

\section{Strengths and weaknesses in relation to other studies}

To our knowledge and despite extensive searching, ${ }^{5}$ there is no literature with which to directly compare our results. Carter ${ }^{14}$ interviewed patients undergoing Bowen therapy for frozen shoulder and very briefly described some experiential aspects of living with the condition, including pain, disturbed sleep, stiffness, impact on mood and a disappointing interaction with a GP and a physiotherapist; all of which accord with our own, more searching, results. But Carter's ${ }^{14}$ main focus was on the experience of the Bowen therapy itself, limiting the applicability of her results and patients' perceptions and priorities-dimensions considered critical by ourselveswere not addressed. Nor are our results directly comparable to those of Hush et $a l^{15}$ who systematically reviewed studies from Canada, the UK, the USA and Scandinavia and found high levels of patient satisfaction with MSK physiotherapy. While our study indirectly encompassed patient satisfaction, it differed in its focus, both in terms of being specific to primary FS and in terms of including experience of the broader care pathway, not physiotherapy alone. Nonetheless, the aspects of care most consistently identified by Hush et $a l^{15}$ as important to patients were the personal and professional attributes of the clinician, explanation and communication of information and treatment outcome. These findings are strongly reinforced by our own work. A consistent international observation is the lack of confidence of GPs in diagnosing subtypes of shoulder pain ${ }^{22}$ and this may have a critical bearing on the appropriateness and timing of care pathways for patients with shoulder conditions.

A key theme from our data was delays in diagnosis. Initially this tended to be a patient-initiated delay resulting from the participants waiting to see if the shoulder issues would resolve. Such patient-initiated delays are not unique to FS and can be seen across a range of health conditions. For example, Solbjør et $a l^{24}$ highlighted that while some of the patients with breast cancer in their study had delayed approaching their GP for 2 weeks, some had waited for longer than 3 months after they had found a lump. When our participants did go to their GP they were often met with a failure to diagnose their condition or they were misdiagnosed. Again, this is not unique to FS; late or missed diagnosis has been cited as a contributory factor in poor outcomes for some cancers. $^{2526}$ Furthermore, Pavey et $a l^{27}$ in their research with patients with motor neurone disease described the long journey to diagnosis as a 'diagnostic quest'.

Following diagnosis, it was evident from our data that FS had a major impact on the lives of our participants. Although FS is not a longer-term chronic condition such as multiple sclerosis or arthritis, it was clear that the participants in our study travelled along a similar trajectory to patients with such conditions. For example, Bury's ${ }^{28}$ notion that chronic health conditions are experienced as a 'biographically disruptive event' resonates very much with the experiences of our participants. Bury ${ }^{28}$ identified the disruption of taken-for-granted behaviours such as general activities of daily living and the disruption of self-concept; all of which are supported by our data.

\section{Implications for clinicians and policymakers}

A care pathway with prompt diagnosis and access to specialist (or informed) care is required for sufferers of FS. There is potential for non-specialist healthcare staff, particularly GPs and physiotherapists, to be made more aware of this condition. This awareness would include diagnostic criteria, expectations, management strategies and patients' needs for information and reassurance.

It is clear that, in some cases, there is a serious mismatch between clinicians' and patients' perceptions of the impact of FS. Strategies for educating clinicians are required, in order to convey the immense impact that frozen shoulder may have on sufferers' lives. Such education should ensure that active measures, to encourage timely resolution, are always offered.

There is a need to provide standardised, consistent information for patients, designed in collaboration with patients and based on the best available evidence. This information should address patients' questions and concerns, rather than clinicians' perceptions of what patients' questions and concerns should be. The information should be available in multiple formats in order to maximise its accessibility.

The Chartered Society of Physiotherapy's (CSP) clinical guidelines for FS and quick reference summary endeavour to address some of these issues. ${ }^{11}{ }^{12}$ Dissemination is now the key to connecting evidence and target audience. Awareness among other healthcare staff should be raised in order that their interaction with this patient group is apposite. 


\section{Future research}

Our participants expressed a clear preference for early specialist referral; also a need for reassurance and timely, comprehensible advice and information in a variety of media. The evidence base underpinning the management of FS is not strong ${ }^{511}$ and studies into the natural history of FS have produced somewhat contradictory results. ${ }^{13}{ }^{29}$ A need for more primary research and for research of higher quality on FS has been identified elsewhere. ${ }^{5}{ }^{11}$ But clinicians could make better use of the evidence, limited though it is.

Until recently, frozen shoulder research has lacked a focus on patients' perspectives. Patient involvement has yet to make appreciable inroads but this must change if such research is to be relevant. Unless the aspects of FS which most concern patients are known, generic shoulder pain patient reported outcome measures (PROMs) - used to measure condition severity and progress in such research, as well as in clinical practice-cannot be considered completely valid. Nor is it possible to develop a more sensitive, condition-specific PROM. ${ }^{30}$ The present study, by exploring which aspects of FS patients most care about, contributes to a foundation for such validation and development.

\section{CONCLUSIONS}

Patients' perspectives on the experience of FS and their priorities for treatment have not previously been explored; however, it is clear that this has been a major omission. Our study identified a number of issues that were important to patients. These included pain, but recovery from functional disability was often given higher priority. Anxiety was another key theme and the struggle of living and dealing with FS was compounded, in some cases, by a lack of awareness on the part of healthcare professionals and, foremost, a failure to diagnose the condition.

To address these issues most effectively it is recommended that a diagnosis, even if only tentative, be quickly established for more patients. This would require GPs to recognise the salient diagnostic features. They and other healthcare workers should also be educated on the condition's impact on individuals and accordingly the findings of the present study should be disseminated and built upon. Advice and information in various formats, reflecting the best available evidence, should be made readily available to patients. Finally, the evidence base for effectiveness of treatments needs to be expanded, maximally utilising patient participation. The present study, by exploring which aspects of FS patients most care about, contributes to this goal.

Acknowledgements Chris Richardson, Heidi Clark, Lorna Goodchild, Jim Robertson, Birgit Hanusch and Lucksy Kottam also contributed by identifying the design, aiding in recruitment and/or commenting on the draft article.

Contributors AR initiated the concept and design, revised the draft and approved the final article; $\mathrm{NH}$ developed the idea for the study with $\mathrm{SH}$ and advised on musculoskeletal and physiotherapy aspects. NH wrote the background section of the article and contributed to the drafting and editing of the whole. SJ conducted the interviews, transcribed and analysed them, wrote up the findings and drafted the body of the article. SH supported SJ, reviewed the analysis, assisted in writing up the findings and contributed to developing and writing the article.

Funding Teesside University Research Fund (application number: 11/10/06)

Competing interests AR reports grants and personal fees from DePuy Ltd, personal fees from JRI Ltd, outside the submitted work; In addition, AR has a patent UK and European pending.

Ethics approval School of Health Research Governance \& Ethics Committee at Teesside University and South Tees Hospitals NHS Foundation Trust Research and Governance Department.

Provenance and peer review Not commissioned; externally peer reviewed.

Data sharing statement Quotes and further details are available from Susan Jones at Susan.Jones@tees.ac.uk

Open Access This is an Open Access article distributed in accordance with the Creative Commons Attribution Non Commercial (CC BY-NC 3.0) license, which permits others to distribute, remix, adapt, build upon this work noncommercially, and license their derivative works on different terms, provided the original work is properly cited and the use is non-commercial. See: http:// creativecommons.org/licenses/by-nc/3.0/

\section{REFERENCES}

1. Hand GCR, Athanasou NA, Matthews T, et al. The pathology of frozen shoulder. J Bone Joint Surg Br 2007;89:928-32.

2. Kelley MJ, McClure PW, Leggin BG. Frozen shoulder: evidence and a proposed model guiding rehabilitation. J Orthop Sports Phys Ther 2009;39:135-48.

3. Robinson CM, Seah KTM, Chee YH, et al. Frozen shoulder. J Bone Joint Surg Br 2012;94:1-9. http://www.ncbi.nlm.nih.gov/pubmed/ 22219239

4. Milgrom $\mathrm{C}$, Novack $\mathrm{V}$, Weil $\mathrm{Y}$, et al. Risk factors for idiopathic frozen shoulder. Isr Med Assoc J 2008;10:361-4.

5. Maund E, Craig D, Suekarran S, et al. Management of frozen shoulder: a systematic review and cost-effectiveness analysis. Health Technol Asses 2012;16:1-243.

6. Walker-Bone K, Palmer KT, Reading I, et al. Prevalence and impact of musculoskeletal disorders of the upper limb in the general population. Arthritis Rheum 2004;51:642-51.

7. Bunker T. Time for a new name for frozen shoulder: contracture of the shoulder. Shoulder Elbow 2009;1:4-9.

8. Shah N, Lewis M. Shoulder adhesive capsulitis: systematic review of randomised trials using multiple corticosteroid injections. $\mathrm{Br} J$ Gen Pract 2007;57:662-7.

9. Boyle-Walker KL, Gabard DL, Bietsch E, et al. A profile of patients with adhesive capsulitis. J Hand Ther 1997;10:222-8.

10. Codman EA. Rupture of the supraspinatus tendon and other lesions in or about the subacromial bursa. Boston: Privately published, 1934.

11. Hanchard N, Goodchild L, Thompson J, et al. Evidence-based clinical guidelines for the diagnosis, assessment and physiotherapy management of contracted (frozen) shoulder. Version 1.7: standard physiotherapy. Endorsed by the Chartered Society of Physiotherapy. 2011. http://www.csp.org.uk/skipp

12. Hanchard NCA, Goodchild L, Thompson J, et al. Evidence-based clinical guidelines for the diagnosis, assessment and physiotherapy management of contracted (frozen) shoulder: quick reference summary. Physiotherapy 2012;98:117-20. http://dx.doi.org/10.1016/ j.physio.2012.01.001

13. Hand C, Clipsham K, Rees JL, et al. Long-term outcome of frozen shoulder. J Shoulder Elbow Surg 2008;17:231-6.

14. Carter B. Clients' experiences of frozen shoulder and its treatment with Bowen technique. Complement Ther Nurs Midwifery 2002;8:204-10.

15. Hush JM, Cameron K, Mackey M. Patient satisfaction with musculoskeletal physical therapy care: a systematic review. Phys Ther 2011;91:25-36.

16. Rookmoneea M, Dennis L, Brealey S, et al. The effectiveness of interventions in the management of patients with primary frozen shoulder. J Bone Joint Surg Br 2010;92:1267-72.

17. Patton MQ. Qualitative research and evaluation methods. 3rd edn. Thousand Oaks, CA: Sage, 2002. 
18. Jack B. Giving them a voice: the value of qualitative research. Nurse Res 2010;17:4-6.

19. Braun V, Clarke V. Using thematic analysis in psychology. Qual Res Psychol 2006;3:77-101.

20. Jones SE, Hamilton S, Perry L, et al. Developing workable research methods: lessons from a pilot study with vulnerable participants and complex assessments. J Res Nurs 2011;16:307-18.

21. Depoy E, Gitlin LN. Introduction to research: understanding and applying multiple strategies. 4th edn. St Louis: Elsevier Mosby, 2005:368.

22. Linsell L, Dawson J, Zondervan K, et al. Prevalence and incidence of adults consulting for shoulder conditions in UK primary care; patterns of diagnosis and referral. Rheumatology (Oxford) 2006;45:215-21.

23. Wofford JL, Mansfield RJ, Watkins RS. Patient characteristics and clinical management of patients with shoulder pain in U.S. primary care settings: secondary data analysis of the National Ambulatory Medical Care Survey. BMC Musculoskelet Dis 2005;6:4. http://www. pubmedcentral.nih.gov/articlerender.fcgi? artid $=549528 \&$ tool $=$ pmcentrez\&rendertype $=$ abstract

24. Solbjør M, Skolbekken J, Sætnan AR, et al. Could screening participation bias symptom interpretation? An interview study on women's interpretations of and responses to cancer symptoms between mammography screening rounds. BMJ Open 2012;2:e001508.

25. Department of Health. Cancer Reform Strategy, 2007. http://www.dh gov.uk/en/Publicationsandstatistics/Publications/

PublicationsPolicyAndGuidance/dh_081006

26. National Patient Safety Agency. Delayed diagnosis of cancer: thematic review 2010. http://www.ntac.nhs.uk/web/FILES/BreastLymphNode/ nhs_1281947508_Delayed_Diagnosis_of_Cancer_in.pdf

27. Pavey A, Allen-Collinson J, Pavey T. The lived experience of diagnosis delivery in motor neurone disease: a sociological-phenomenological study. Sociol Rese Online 2013;18:11. http://www.socresonline.org.uk/18/2/11.html

28. Bury MR. Chronic illness as biographical disruption. Sociol Health III 1982;4:167-82.

29. Binder Al, Bulgen DY, Hazleman BL, et al. Frozen shoulder: a long-term prospective study. Ann Rheum Dis 1984;43: 361-4.

30. Department of Health. Guidance on the routine collection of Patient Reported Outcome Measures (PROMs). 2008. http://www.mstrust. org.uk/competencies/downloads/NHS-PROMS.pdf 